\title{
REVIEW
}

\section{CFTR biomarkers: time for promotion to}

\section{surrogate end-point?}

\author{
K. De Boeck, L. Kent, J. Davies, N. Derichs, M. Amaral, S.M. Rowe, P. Middleton, \\ H. de Jonge, I. Bronsveld, M. Wilschanski, P. Melotti, I. Danner-Boucher, S. Boerner, \\ I. Fajac, K. Southern, R.A. de Nooijer, A. Bot, Y. de Rijke, E. de Wachter, T. Leal, \\ F. Vermeulen, M.J. Hug, G. Rault, T. Nguyen-Khoa, C. Barreto, M. Proesmans and \\ I. Sermet-Gaudelus on behalf of the European Cystic Fibrosis Society Clinical Trial \\ Network Standardisation Committee
}

ABSTRACT: In patients with cystic fibrosis, cystic fibrosis transmembrane conductance regulator (CFTR) biomarkers, such as sweat chloride concentration and/or nasal potential difference, are used as end-points of efficacy in phase-III clinical trials with the disease modifying drugs ivacaftor (VX-770), VX809 and ataluren. The aim of this project was to review the literature on reliability, validity and responsiveness of nasal potential difference, sweat chloride and intestinal current measurement in patients with cystic fibrosis.

Data on clinimetric properties were collected for each biomarker and reviewed by an international team of experts. Data on reliability, validity and responsiveness were tabulated. In addition, narrative answers to four key questions were discussed and agreed by the team of experts.

The data collected demonstrated the reliability, validity and responsiveness of nasal potential difference. Fewer data were found on reliability of sweat chloride concentration; however, validity and responsiveness were demonstrated. Validity was demonstrated for intestinal current measurement, but further information is required on reliability and responsiveness. For all three end-points, normal values were collected and further research requirements were proposed.

This body of work adds useful information to support the promotion of CFTR biomarkers to surrogate end-points and to guide further research in the area.

KEYWORDS: Clinical trials, cystic fibrosis transmembrane conductance regulator, intestinal current measurement, nasal potential difference, surrogate end-point, sweat test

O utcome measures fall into three classes: clinical end-points, surrogate end-points and biomarkers.

Clinical end-points reflect how a patient feels, functions or survives [1,2] and detect a tangible benefit for the patient. The improved life expectancy in cystic fibrosis has rendered survival, the gold standard clinical efficacy measure, an impossible end-point to use in clinical trials. Therefore, intermediate clinical efficacy measures, such as the frequency of respiratory exacerbation were introduced. The latter has been used in registration trials for rhDNase [3], tobramycin solution for inhalation [4] and aztreonam lysinate [5]. Clinical end-points particularly useful for young children include anthropometric measures. Quality of life as measured by the Cystic Fibrosis QuestionnaireRevised is also accepted as a measure of treatment benefit in cystic fibrosis [6, 7]; however, it is considered only an optional end-point by the European Medicines Agency $[6,8]$.

A surrogate end-point is a laboratory measurement used as a substitute for clinical end-points and predicts the efficacy or toxicity of therapy [1, 2]. It is an indirect measurement of effect and is used when direct measurement of clinical effect is not feasible or practical. Surrogate end-points can be used complementary to measures of treatment
AFFILIATIONS

For a full list of author affiliations, please refer to the

Acknowledgements section.

CORRESPONDENCE

K. De Boeck

University Hospital

Gasthuisberg

CF Reference Centre

Herestraat 49

3000 Leuven

Belgium

E-mail: Christiane.deboeck@

uzleuven.be

Received:

April 062012

Accepted:

April 112012

First published online:

Aug 092012 
benefit and may shorten the period of follow-up required. The link between the surrogate end-point and survival, long-term prognosis or accepted measures of treatment effect (both improvement and deterioration) must be proven. Forced expiratory volume in $1 \mathrm{~s}$ (FEV1) has been widely used as a surrogate end-point due to the established link with survival [9]. However, in many patients with cystic fibrosis, the rate of decline in FEV1 has slowed [10], limiting the current sensitivity of the measure, particularly in children or in patients with mild lung disease $[10,11]$.

A biomarker is defined as "a characteristic that is objectively measured and evaluated as an indicator of normal biologic processes, pathogenic processes or pharmacologic response to a therapeutic intervention" (e.g. nasal potential difference (NPD), mucociliary clearance, inflammatory markers and sputum bacterial density) [1, 2]. These measures are mainly used in phase-I or -II clinical trials when proof-of-concept for a specific compound is explored. Biomarkers are useful for gaining information about the mechanism of action of potential drugs, for identifying treatment responders and for dose selection. Some biomarkers are currently being considered for "promotion" to the status of surrogate endpoint. They are often used as secondary outcome measures in phase-III trials which provide data on responsiveness, confirm mechanism of action and compile information for promotion of biomarkers to surrogate outcome measure. During phase-III trials with ivacaftor, cystic fibrosis transmembrane conductance regulator (CFTR) correctors (Vertex Pharmaceuticals Ltd, Cambridge, MA, USA) and ataluren (PTC Therapeutics Inc., South Plainfield, NJ, USA) in patients with cystic fibrosis, CFTR biomarkers are being used as end-points. These new therapies address the basic defect in cystic fibrosis and may be particularly well suited for people with early or mild lung disease.

To gain acceptance by researchers and licensing bodies, an outcome measure must be assessed for clinimetric properties, such as reliability, validity and responsiveness to treatment (table 1). Reliability (e.g. an assessment of the consistency of a given measurement), is important, both in terms of inherent biological variation (repeatability) and also in relation to differences across different assessors and centres (reproducibility). To optimise reliability in multiple centre trials, standardised operating procedures (SOPs) and training are needed [12]. Validity refers to clinical and biological relevance; in other words, there must be a direct link with the disease process and the mechanism of action of the intervention [13]. The outcome measure should correlate with established measures of treatment benefit or a gold standard (i.e. concurrent validity and predictive validity) and reflect clinical severity (i.e. discriminate validity) [14]. When a gold standard is not available, evaluation of convergent validity can be performed (i.e. an outcome measure can be compared with another which measures the same attribute). Prediction of prognosis is also important, for example, the ability to predict survival (predictive validity). Responsiveness refers to the ability of the measurement to detect change due to an intervention known to alter the attribute of interest.

Also important in the development of surrogate end-points is feasibility, referring to financial, practical and ethical considerations, as well as patient and assessor acceptability [15]. A feasible end-point should be cost-effective, pose minimal risk/ discomfort to the patient and should be applicable throughout the entire range of ages and disease severities. Feasibility will determine whether outcome measures gain acceptance into research practice. Clinimetric properties and feasibility are population and situation dependent.

\section{SCOPE AND PURPOSE OF THE GUIDELINE}

This guideline documents the European Cystic Fibrosis Society (ECFS) Clinical Trial Network's current agreement on aspects of CFTR biomarkers for use in clinical trials in the area of cystic fibrosis. After preparatory work over a period of 6 months, participants met twice to discuss their results and conclusions (November 17 and 18, 2010, and June 9, 2011). This resulted in a draft document that was circulated among all participants and further amended.

After a description of the CFTR biomarkers, we explore the clinimetrics and the feasibility of the chosen outcome measures, we report on their use in clinical trials and we conclude by answering the following questions. 1) Do CFTR bio-assays have the potential to become surrogate outcomes? 2) For what kind of therapeutic trial is this outcome appropriate (therapeutic aim, phase of trial, target population, trial duration, number of patients involved and number of sites involved)? 3) Within what time frame can change be expected and what treatment effect can be considered clinically significant? 4) What are the most needed studies to further define these outcome measures in patients with cystic fibrosis?

The guideline also provides an inventory of the literature on selected CFTR bio-assays. We chose to include papers published since 1980 only. It is hoped that this document will offer some guidance for pharmaceutical companies, investigators and regulatory authorities.

\section{CFTR BIO-ASSAYS}

CFTR biomarkers measure the presence and/or function of the CFTR protein in different organs. We chose to discuss the sweat chloride test, NPD measurements and intestinal current measurements (ICMs) because they are functional assays and not only document the presence of CFTR, but also its ion transport activity. As such, they are most appropriate for use in clinical trials of compounds aiming to correct the basic defect in patients with cystic fibrosis, e.g. gene therapy and small molecules such as CFTR potentiators, correctors, and premature termination codon suppressors [16-21]. These biomarkers of CFTR function are currently used to confirm the diagnosis of cystic fibrosis [22-25]. Since values for these biomarkers differ in cystic fibrosis versus non-cystic fibrosis subjects, it seems logical to hypothesise that, when treatments correct the basic CFTR defect at the protein level, the values for these biomarkers will change as well.

After stimulation of sweat production by pilocarpine iontophoresis and collection of sweat in a gauze or collector (Macroduct ${ }^{\circledR}$; Wescor Inc., Logan, NV, USA), the sweat chloride concentration is determined by original titration with colorimetric end-point, by titration with coulometric end-point (chloridometer), by in situ selective electrode (Exsudose ${ }^{\circledR}$; TemSega, Lormont, France) or by indirect potentiometry [26]. The increase in sweat chloride concentration in cystic fibrosis is the consequence of decreased chloride re-absorption via CFTR in the water impermeable sweat ducts [27]. NPD and ICM measure the voltage potential or electrical current, respectively, resulting from epithelial ion fluxes 


\section{TABLE 1 Definitions and justification of importance for clinimetric/psychometric properties}

Clinimetric/
psychometric property $\begin{aligned} & \text { Definition } \\ & \text { Regree to which a measurement is consistent and free } \\ & \text { from error } \\ & \text { Concurrent validity: degree to which a test correlates } \\ & \text { with a "gold standard" criterion test which has been } \\ & \text { established as a valid test of the attribute of interest } \\ & \text { Convergent validity: degree to which a test correlates } \\ & \text { with another test which measures the same attribute } \\ & \text { Discriminate validity: degree to which a test differentiates } \\ & \text { between groups of individuals known to differ in the } \\ & \text { attribute of interest } \\ & \text { Predictive validity: degree to which an attribute can } \\ & \text { be predicted using the result of a predictor test/or } \\ & \text { degree to which prognosis can be predicted } \\ & \text { Degree to which a test changes in response to an } \\ & \text { intervention known to alter the attribute of interest }\end{aligned}$

Justification of importance

Important to quantify error (systematic and random) so that true changes can be discerned from changes due to normal fluctuations

The gold standard outcome measures are often not feasible; therefore, it is important to know how an alternative outcome measure compares to the gold standard, and how different outcome measures compare It is important to know the ability of outcome measures to discriminate between different groups

Important attribute of tests used in clinical practice or research to assess treatment benefit (e.g. to identify improvements response to an intervention) at the mucosal surface in vivo and ex vivo, respectively. The NPD measurement is thought to provide information on both sodium absorption and chloride secretion [28, 29]. In normal airway epithelia, sodium absorption is the primary ion transport activity so that the resulting airway surface potential difference is negative with reference to the interstitium. Perfusion of the ENaC channel blocker amiloride will lead to a less negative potential difference. Creating a chemical gradient for chloride by superfusion of chloride free solution followed by activation of the CFTR channel with isoproterenol, will lead to chloride secretion and thus again a more negative potential difference. In contrast, in cystic fibrosis subjects there is heightened $\mathrm{ENaC}$ mediated sodium absorption due to absent or dysfunctional CFTR [30-32]. The resultant baseline potential difference is thus more negative. The change with application of amiloride is larger, whereas minimal or no change in potential difference is seen upon stimulation of chloride secretion through CFTR dependent pathways. Recently the notion of increased sodium absorption in cystic fibrosis epithelia has been challenged by data in the newborn cystic fibrosis pig and in cultured tracheal epithelia $[33,34]$. In these models, the defective CFTR chloride current seemed sufficient to explain all phases of the NPD measurement. For ICM, an intestinal (usually rectal suction) biopsy and special micro-Ussing chamber are needed for measurement of ex vivo transepithelial short-circuit current (Isc) as a measure of net ion fluxes across the tissue. In cystic fibrosis, the intestinal CFTR-mediated chloride secretion is impaired, while absorptive processes remain unchanged or may be enhanced. In cystic fibrosis, the normal Isc response to forskolin, an activator of CFTR, is absent or reduced. The Isc responses to carbachol and histamine consist of two components: a lumen-positive current that is most likely caused by the apical potassium efflux, and a lumen-negative current, caused by apical chloride secretion. In ICM of healthy individuals, the apical potassium efflux in reaction to carbachol and histamine is masked by the much larger chloride efflux. In cystic fibrosis, the response is reversed due to the apical potassium efflux in the absence of a chloride efflux, or biphasic due to residual CFTR-mediated chloride efflux in milder forms of cystic fibrosis [35-37].

\section{CLINIMETRICS OF CFTR BIO-ASSAYS}

For NPD, data were collected on reliability (table 2), validity (table S1, online supplement) and responsiveness (table 3). Eight studies document reliability of NPD and demonstrate that with repeated measurements, the mean results per group and the diagnostic conclusions do not differ; however, the within-subject variability is considerable. There is strong evidence that NPD has excellent discrimination validity. 25 studies consistently show a statistically significant difference in chloride and sodium conductance between patients with cystic fibrosis and healthy controls. In patients with "questionable" cystic fibrosis, NPD composite scores provided a highly sensitive tool to diagnose patients as "CF-likely" and "CF-unlikely", with both cohorts having significantly different disease presentation [39, 77-79]. Data from studies with ataluren, ivacaftor, the CFTR corrector VX-809 and gene therapy confirm that NPD is a responsive endpoint. In the earliest gene therapy trials, the overall results were not uniformly conclusive. The low subject numbers of subjects along with the relatively low bioactivity of the agents tested may explain these non-significant results for NPD. Tables S2 and S3 in the online supplement report reference values for NPD measures in patients with cystic fibrosis and in healthy controls. The majority of the available data concerns adults.

Reliability data for sweat chloride are inconclusive as these are mainly from retrospective studies with few, or combined cystic fibrosis and non-cystic fibrosis individuals (table S4, online supplement). Data clearly establish validity of sweat chloride, which discriminates between patients with cystic fibrosis and non-cystic fibrosis individuals, between patients with cystic fibrosis and carriers (table S5, online supplement) and between patients with different disease severity (e.g. patients with pancreatic sufficiency and insufficiency). Individuals grouped according to their sweat chloride result had significantly different disease presentation. The sweat chloride concentration has been used as an end-point in studies of ivacaftor and VX-809 which clearly demonstrated responsiveness of this parameter (table 3). However, one study investigating ataluren demonstrated a 


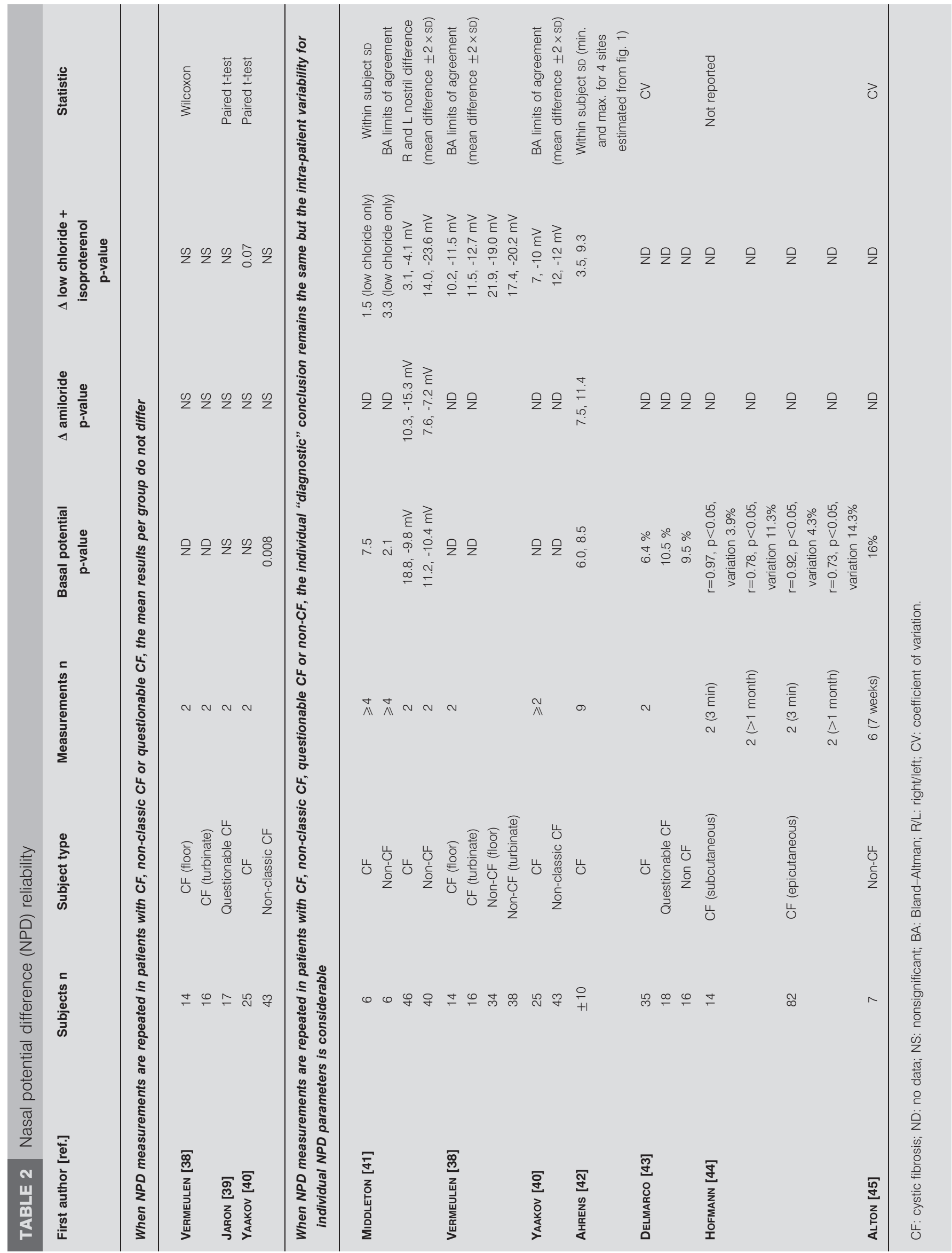




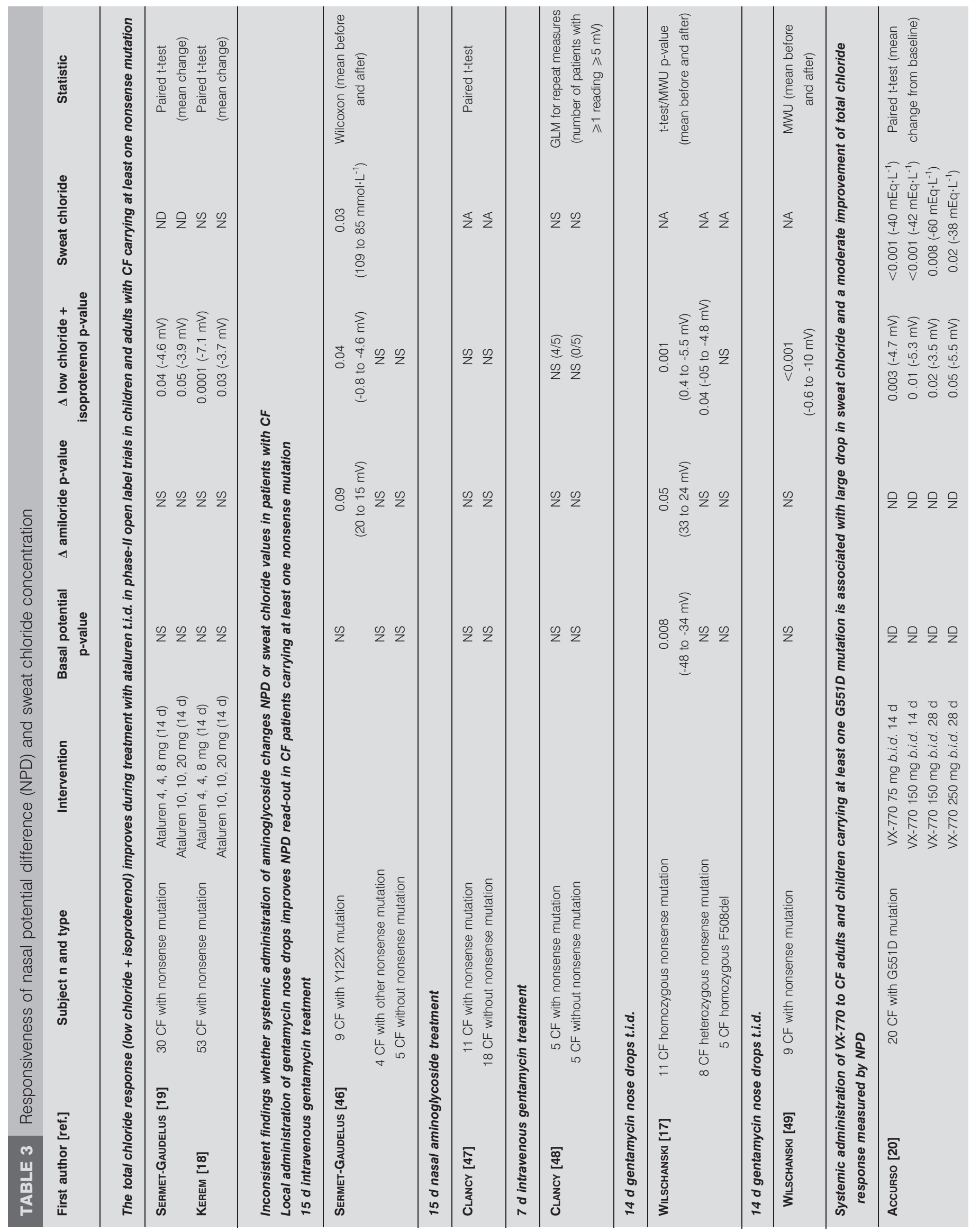




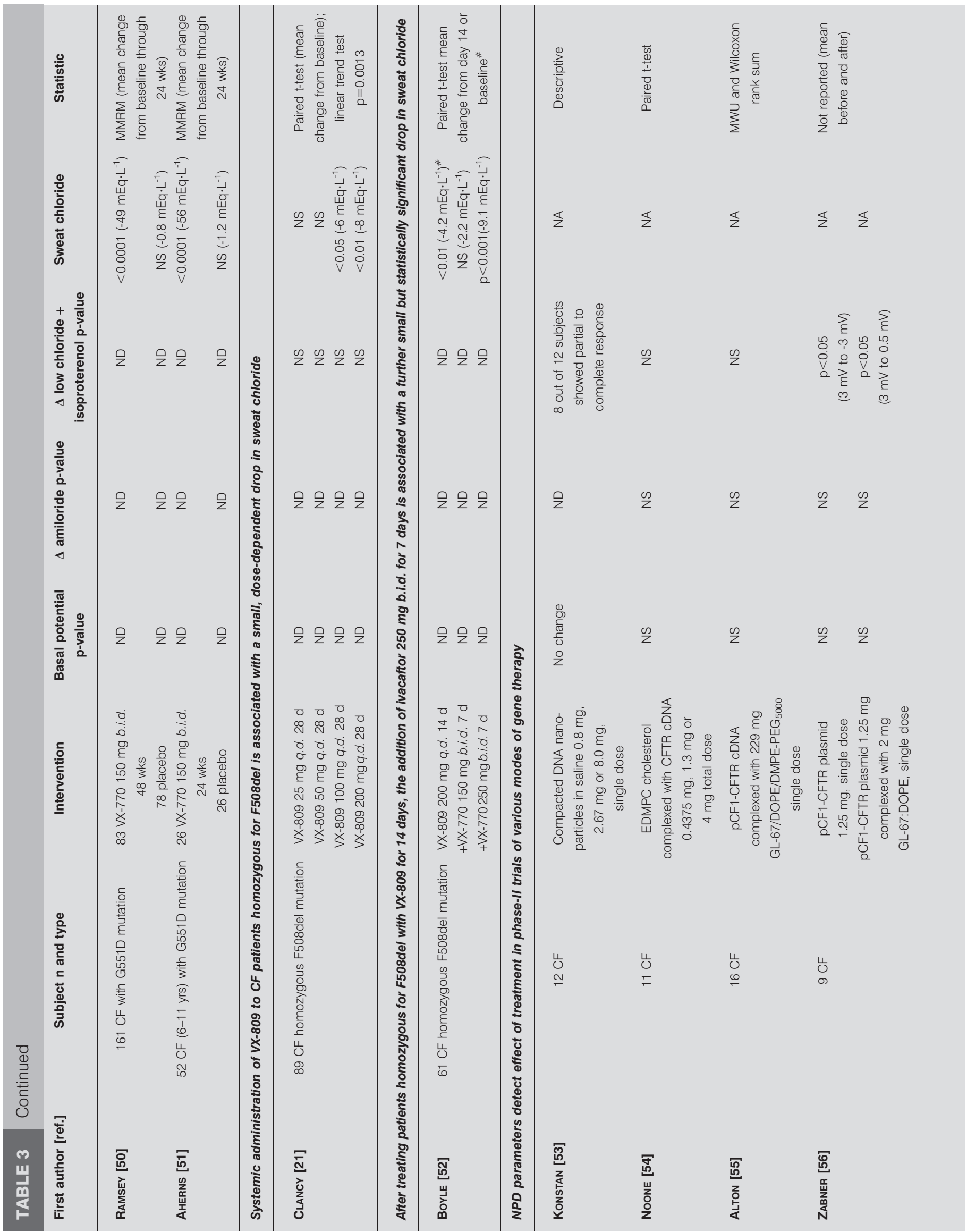




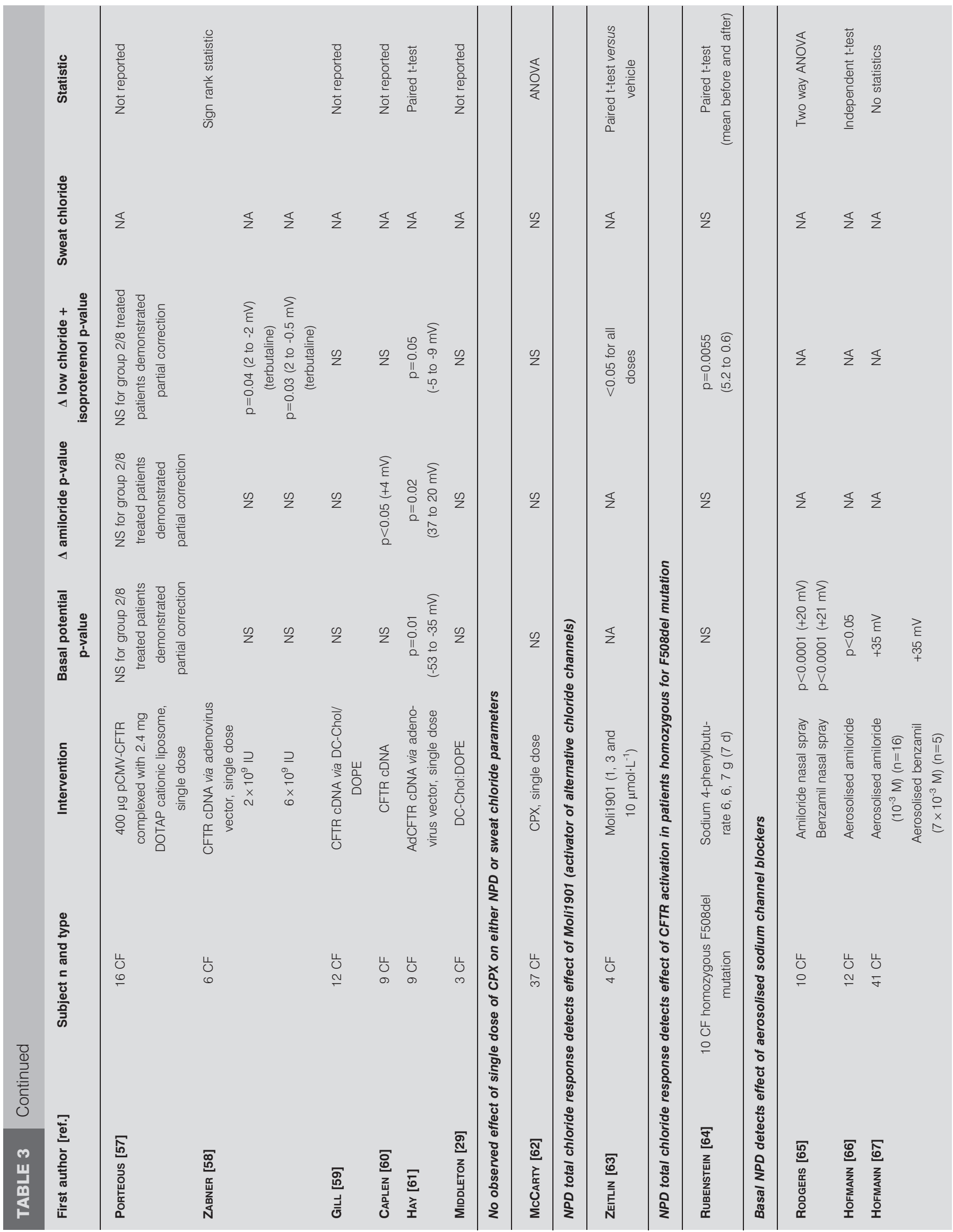




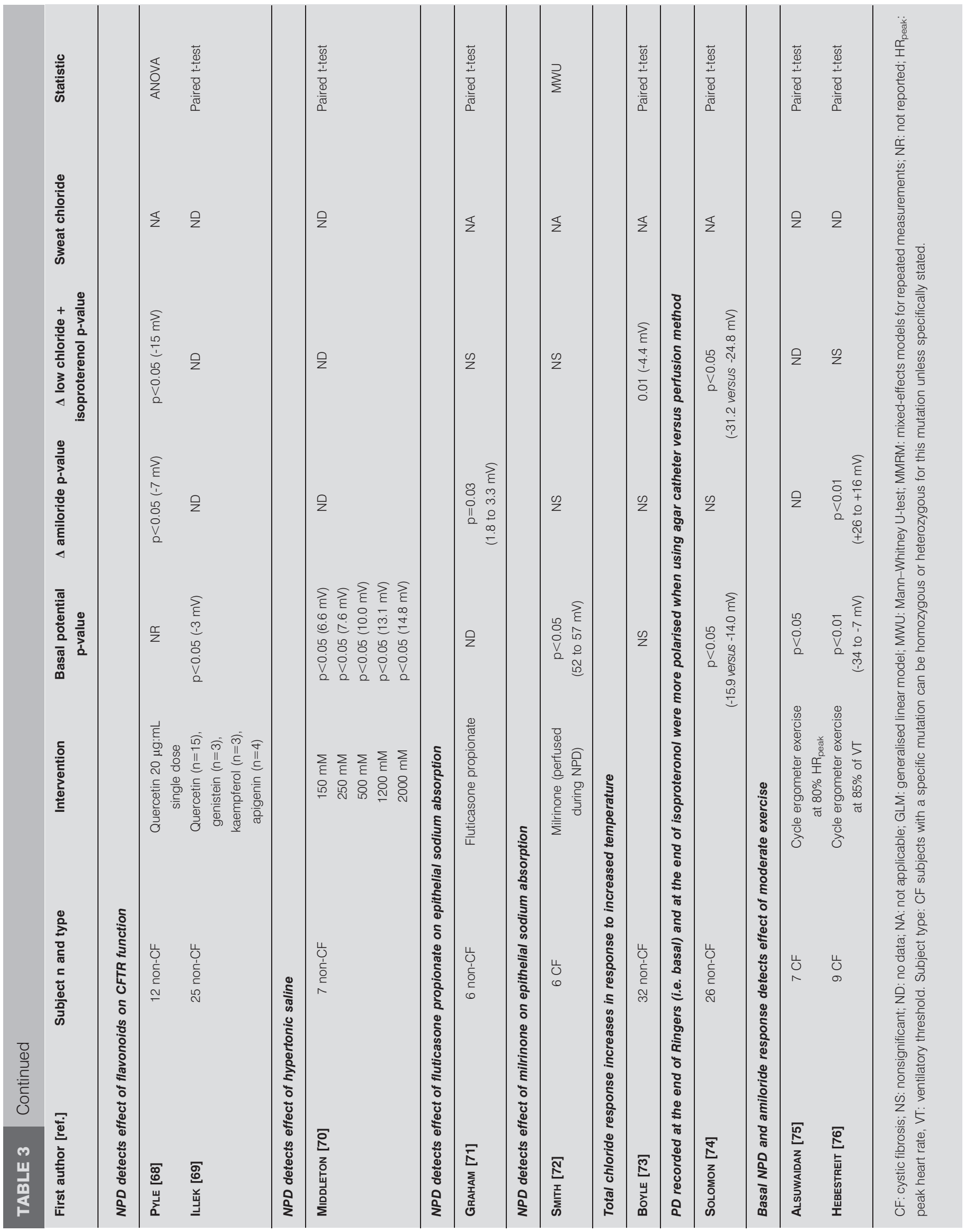


significant difference in NPD but failed to show a difference in sweat chloride [18]. Subsequently, the sweat chloride test was not included as an end-point in additional phase-II trials of ataluren, but is currently being evaluated in the phase-III trial.

Few studies were found of clinimetric properties of ICM (table S6, online supplement). No studies were found on reliability. ICM has been shown to discriminate between patients with cystic fibrosis and healthy individuals [37, 80-86] and, at a group level, can discriminate between pancreatic sufficient and insufficient patients [35]. Similar to the sweat test and the NPD, patients with cystic fibrosis who were grouped according to their ICM result have been shown to differ in disease presentation: the more chloride secretion measured in the rectal mucosa, the milder the disease presentation $[35,37,82]$. These data provide evidence of sound discriminate validity. ICM has been shown to correlate well with results from CFTR mutation analysis and moderately with sweat chloride [37]. No studies used ICM as an end-point. For reference values we refer to DeRICHS et al. [37].

\section{FEASIBILITY OF CFTR BIO-ASSAYS}

The sweat test has a long tradition and is widely available, relatively noninvasive and easy to perform, but for reliable performance rigorous adherence to standard techniques is needed $[26,87,88]$. The more recent measurements of NPD and ICM are limited to selected centres with expertise. Given the complexity of these tests, strict adherence to SOPs is important.

All three tests can be performed from infancy through to adulthood. However, NPD can be problematic in young children. NPD in infants can be done for diagnostic purposes in single centres with extensive experience in this age group $[77,89]$. As such, use of NPD as an outcome measure in clinical trials in infants and preschool children has a limited role. Conversely, ICM may be better tolerated by younger children than in adults because it involves rectal sampling. Obtaining a sufficient amount of sweat can be an issue in some (mainly young) patients. Obtaining valid NPD measurements may be impossible or (temporarily) unreliable in subjects with acute upper respiratory tract infection, extensive nasal polyps or after prior sinus surgery.

The risk of infection is minimal in all three tests when care is taken to discard, disinfect or sterilise equipment as appropriate. Electrical equipment for sweat testing and NPD should be checked annually for current control and leakage. The sweat test is viewed as comfortable and very safe as it uses a low voltage electrical current produced by a battery. Some local erythema lasting a few hours is expected; skin burns can occur when sweat test equipment is not properly handled [26]. A small scab or skin scar can occur when too deep a skin abrasion is performed during NPD measurement. Rarely, a rectal bleed can occur after biopsy taking for ICM [90] which is contraindicated in patients with abnormal haemostasis or portal hypertension.

The cost for equipment is lower for the sweat test than for the NPD or ICM. The sweat test requires staff time to cover sampling and assay. The NPD requires staff time to prepare solutions and catheters/bridges and to perform the procedure. ICM requires an experienced gastroenterologist/cystic fibrosis specialist to obtain the sample, a research nurse and a technician. The time required to perform each test is approximately the same (90$120 \mathrm{~min})$. The sweat test and ICM require clinical space for sample collection and laboratory space for assay. NPD requires sufficient clinical space to accommodate the equipment along with the personnel due to the in vivo nature of the test.

Training is required to perform each test. Dedicated laboratory personnel can easily learn sweat collection and analysis assay. NPD and ICM require more extensive training and experience in order to minimise variability of the results (for NPD, correct placement and fixation of the catheter, real time interpretation of readings, including stable baseline and end of response to solutions, and troubleshooting; for ICM, biopsy taking, mounting the tissue in the Ussing chamber, real time interpretation of readings and checking biopsy viability).

\section{COMPARISON OF THE DIFFERENT CFTR BIOMARKERS}

The advantages of using sweat chloride as outcome measure are its feasibility, availability and the assessment of CFTR function in an organ unaffected by chronic infection and inflammation. Results evaluating ivacaftor and VX-809 also suggest it is more sensitive to small changes in CFTR activity. The advantage of NPD is that it reflects CFTR function in the respiratory tract (albeit the upper respiratory tract), the organ strongly related to cystic fibrosis survival. Measurements in the lower respiratory tract can be performed bronchoscopically [91] but are too complex and invasive for use in large scale trials. Advantages of ICM include easy application in young children and the ability to measure both chloride and bicarbonate transport. It is anticipated that ICM may have a fast response to CFTR correctors because of the exceptionally high cell turnover in the intestinal epithelium that renews itself within 3-5 days).

Limitations of sweat testing and ICM include that they do not measure CFTR activity in the respiratory epithelium. Limitations of NPD include the large intra-subject variability and the difficulty of performing it in young children. Although it is a painless procedure, some adults are reluctant to undergo a rectal suction biopsy. Other limitations of the ICM are the very low number of centres with expertise and the short viability of the rectal biopsies, which precludes long-distance transport to a central laboratory for analysis.

\section{USE OF SWEAT TEST, NPD AND ICM AS OUTCOME PARAMETERS IN CLINICAL TRIALS TO DATE}

Sweat chloride is an appropriate biomarker in clinical trials for systemic therapies only. Marked changes in sweat chloride occurred after administration of the CFTR potentiator ivacaftor to cystic fibrosis subjects with the G551D mutation [20, 50]. In subjects homozygous for the F508del mutation, small changes were seen after intervention with the CFTR corrector VX-809 [21] and moderate changes during combination treatment with ivacaftor and VX-809 [52]. In patients with a nonsense mutation, ataluren improved NPD but not sweat chloride [18, 19]. Therefore, the organ specificity or efficacy might differ between drugs.

In CFTR gene therapy trials, applications of viral and synthetic vectors to the nasal epithelium have resulted in significant changes in chloride secretion on NPD. Interventions with the nonsense mutation read-through drugs (aminoglycosides and ataluren) [17-19] have also been proven to change only the chloride response and not the basal potential nor amiloride response. An improvement in chloride and sodium transport was observed with ivacaftor therapy, the latter only in the 
combined data set from the two parts of the trial [20]. In patients exposed to ataluren for 84 days, both components of the total chloride response, the zero chloride response and the isoproterenol response, improved significantly, but the zero chloride improvement was larger [92]. In the ivacaftor trial where both have been measured, changes in sweat chloride concentration were more impressive than changes in NPD readout [20].

For ICM, substantial experience has been gathered in preclinical human ex vivo corrector studies $[93,94]$. What follows can be taken into consideration when contemplating use of ICM as an outcome parameter. CFTR is the dominant, if not sole, apical chloride channel in the intestine and becomes ratelimiting for transepithelial chloride transport in rectal biopsies at CFTR protein levels below $\sim 20 \%$ of wild-type controls. Therefore, a small gain in CFTR expression or function induced by CFTR corrector compounds (e.g. from 1\% to 5\% of wild-type values) will result in a large gain in chloride and bicarbonate secretory current (Isc) (e.g. from 5 to $25 \%$ of wild-type controls). In contrast to the sweat test and to NPD, ICM performed with bicarbonate rich perfusion fluid provides information on CFTRdependent bicarbonate secretion, an important and cystic fibrosis-relevant determinant of mucus release, expansion and viscosity [95]. ICM is the only biomarker that can directly assess the beneficial effects of pure CFTR correctors, i.e. compounds that allow the mutant F508del-CFTR to reach the plasma membrane [96]. Rescued F508del-CFTR has major gating defects [97] that might be overcome by CFTR potentiators, i.e. compounds that increase the opening of the CFTR channel. Since ICM evaluates CFTR activity ex vivo, potent potentiators (e.g. genistein and ivacaftor) can be applied directly on the tissue removed from the patient under corrector treatment to assess full CFTR activity and hence membrane rescue of the mutant protein. When using sweat test or NPD as outcome measure, pure correctors can only be tested properly in vivo by conducting combination trials of correctors with potentiators to overcome the gating defect of the rescued mutant protein [52].

\section{QUESTION 1: DO SWEAT CHLORIDE, NPD AND ICM HAVE THE POTENTIAL TO BECOME SURROGATE OUTCOMES?}

Our view is that each of these measures has the potential to be a surrogate outcome since they are in vivo (sweat chloride and NPD) and ex vivo (ICM) markers of CFTR function. To achieve this, long-term studies with disease modifying drugs need to demonstrate that improvement in CFTR function correlates with improvement in clinically relevant outcomes (increased longevity, patient reported outcomes and decrease in pulmonary exacerbations) or surrogate outcomes (improvement in FEV1). In patients aged 12 yrs and older, treatment with ivacaftor for 48 weeks led to large improvements in sweat chloride and clinical as well as surrogate outcome measures: a decrease of sweat chloride concentration from a mean of $100 \mathrm{mmol} \cdot \mathrm{L}^{-1}$ to below $60 \mathrm{mmol} \cdot \mathrm{L}^{-1}$, a mean weight gain of $3.1 \mathrm{~kg}$, a $55 \%$ decrease in likelihood of experiencing a pulmonary exacerbation and a mean improvement of $10.6 \%$ predicted in FEV1 [50]. The intermediate results of the ivacaftor trial in 52 children 611 yrs of age demonstrate the same improvement in all outcomes: a drop in sweat chloride concentration from a mean of 104 to $60 \mathrm{mmol} \cdot \mathrm{L}^{-1}$, a large weight gain, and a mean improvement in FEV1 of $12.7 \%$ pred or $17.4 \%$ change from baseline [51]. Concurrent overall changes in the clinical outcome, surrogate outcome and the sweat test result are expected, rather than a close correlation between the improvement in sweat chloride concentration and the improvement in clinical or surrogate outcome. Indeed, the latter are dependent on many variables (including those unrelated to disease mechanism, such as environment, adherence, and exposure to respiratory infections). The ongoing phase-III ataluren trial is expected to provide additional information, and may offer further data supporting the use of sweat test and NPD as surrogate outcome measures.

\section{QUESTION 2: FOR WHAT KIND OF THERAPEUTIC TRIAL ARE CFTR BIO-ASSAYS APPROPRIATE (THERAPEUTIC AIM, PHASE OF TRIAL, TARGET POPULATION, TRIAL DURATION, NUMBER OF PATIENTS INVOLVED AND NUMBER OF SITES INVOLVED)?}

Sweat chloride concentration and NPD are particularly well suited for phase-II trials with disease modifying therapies aimed at correcting the basic CFTR defect via gene therapy or strategies to rescue or potentiate CFTR protein. Power calculations need to take into consideration the moderate (sweat chloride concentration) to large (NPD) intra-subject variability (for specific values consult the online table) and the uncertainty of the effect size that should be aimed for (see further). For phase-III studies involving systemic drugs, sweat chloride concentration may be the most feasible choice. Given the complexity of the NPD technique and the large intra-subject variability even in sites with great expertise, a large, multicentre trial using NPD as outcome can be challenging and costly, but is presently in progress for the ataluren phase-III trial.

For similar therapies, ICM may be useful in phase-II clinical trials in adults, children and infants with cystic fibrosis. But more information on reliability is required before firm statements can be made. ICM has, at present, most application in preclinical drug testing of potentiators and correctors. As stated above, for "pure corrector compounds", only ICM is appropriate.

Cystic fibrosis is a rare disease with at present a slow lung disease progression, especially in young patients. This makes demonstration of real clinical benefit in phase-III studies extremely difficult in children. Therefore, sweat chloride and NPD, being in the causal pathway of the disease, could be considered as efficacy outcome measures in such phase-III trials with disease modifying drugs, especially if a compound has proven efficacy and safety in adults. Efficacy and further safety testing can follow during phase-IV pharmacovigilance. Using a biomarker or surrogate outcome as preliminary proof of efficacy is also suggested in the European Medicines Agency guidance for trials in small populations [98, 99].

\section{QUESTION 3: WITHIN WHAT TIME FRAME CAN CHANGE BE EXPECTED AND WHAT TREATMENT EFFECT CAN BE CONSIDERED CLINICALLY SIGNIFICANT?}

The timeline in which changes in the measurement will be detected will depend on the mechanism of action of the drug and on the rate of renewal of the epithelium studied. The kinetics of such changes in humans have not been widely evaluated. During treatment with the potentiator ivacaftor, improvements in sweat chloride concentration and NPD have been demonstrated at the earliest time point measured ( 3 days and 14 days, respectively) [20]. During treatment with the CFTR 
corrector VX-809, alone or in combination with the potentiator ivacaftor, decreases in sweat chloride concentration were reported at day 14 to 21 [100]. Also during treatment with ataluren, changes in NPD readout were present at the first time point measured, i.e. 14 days [18, 19].

The magnitude of change which is of clinical significance has not been established for any of the CFTR bio-assays. The mean changes in sweat chloride concentration reported with ivacaftor were large (in the order of 50-60 $\mathrm{mmol} \cdot \mathrm{L}^{-1}$ ) [20, 50, 51]. Since, in these trials, all clinical and surrogate outcomes improved, one can conclude that such changes in sweat chloride are clinically meaningful. Further analysis of these data may help to determine if a cut-off value of improvement in sweat chloride concentration can be correlated to a change in clinical benefit. Determining the minimally clinically important difference will be an important parameter for guiding the development of further agents active towards modulating CFTR. A zero chloride plus isoproterenol response above the threshold of -5 to $-7 \mathrm{mV}$ is considered significant because it is the cut-off between cystic fibrosis and non-cystic fibrosis subjects in cross sectional evaluation. Prospective phase-III studies still have to provide evidence for this assumption. To assess response in an individual, the correct approach may be to monitor whether a repeated test, measured to monitor the response to an intervention, has changed beyond its natural variability [101]. In the phase-II ivacaftor trial, the improvement in NPD chloride secretion was small, i.e. only $-3.5 \mathrm{mV}$ [20]; still, the clinical benefit of this drug is very marked. In another trial, small sweat chloride changes were detected with VX-809 therapy [21], whereas no changes in NPD or lung function was observed. Therefore, the relative sensitivity of changes in different outcomes is at present unclear. Is NPD less sensitive than sweat test? Will a CFTR measurement in the respiratory tract give a better prediction of respiratory outcome than, for example, the sweat test? Will modifier drugs differ in their organ specific efficacies? For NPD we need to keep in mind that changes in basal $\mathrm{PD}$ and changes in amiloride response reflect sodium transport, whereas changes in zero chloride and isoproterenol response reflect chloride transport. It remains to be determined which of these is most important for disease amelioration.

Only theoretical considerations can be made regarding ICM. Because of the fast renewal rate of intestinal epithelium (3-5 days), test compounds that act by improving CFTR function through effects on de novo protein synthesis are expected to show full beneficial effects in less than 1 week, abolishing the need for prolonged testing.

\section{QUESTION 4: WHAT ARE THE MOST NEEDED STUDIES TO FURTHER DEFINE THIS OUTCOME MEASURE IN PATIENTS WITH CYSTIC FIBROSIS?}

For sweat test, better knowledge of reliability in genetically well-defined controls and cystic fibrosis patients is needed. For NPD and ICM, further unification of test performance and establishment of normal values for use in multicentre trials are needed. These aims are being addressed by the new ECFS NPD and ICM SOPs and the ongoing multicentre reference data validation study in the ECFS Diagnostic Network Working Group. In addition, the track record of these biomarkers in longitudinal phase-III studies is needed. We must understand which change in CFTR bio-assay is associated with long-term clinical benefit of drug therapy, and how well this associates in individual responses.

\section{CONCLUSION}

This document provides a systematic review of the clinimetric properties of CFTR biomarkers and provides supporting evidence for promoting these biomarkers to surrogate endpoints. Data collected demonstrate the reliability, validity and responsiveness of NPD. Fewer data were found on reliability of sweat chloride concentration; however, validity and responsiveness are demonstrated. Validity is demonstrated for ICM, but further information is required on reliability and responsiveness. Normal values are collected for all three end-points. Further research requirements are proposed for each end-point. In particular, sweat test and ICM require further supporting data.

There is great interest in biomarkers and surrogate end-points in cystic fibrosis. It is already more than a decade ago that participants in a National Institutes of Health (NIH) workshop challenged statisticians to develop robust metrics to study relationships between surrogate end-points, clinical end-points and interventions [1]. That NIH workshop also highlighted the need to assess data from both epidemiological studies and randomised clinical trials as a source of information on biomarkers when considering promotion to surrogacy [1]. In a small population such as cystic fibrosis, it is all the more important that valuable information is shared and that centres work together to improve clinical research.

\section{STATEMENT OF INTEREST}

None declared.

\section{ACKNOWLEDGEMENTS}

Author affiliations are as follows. K. De Boeck, F. Vermeulen and M. Proesmans: University Hospital of Leuven, Leuven, Belgium; L. Kent: University of Ulster, and Belfast Health and Social Care Trust, Belfast, UK; J. Davies: Dept of Gene Therapy, National Heart and Lung Institute, Imperial College, London, UK; N. Derichs: CFTR Biomarker Centre and Cystic Fibrosis Centre, Dept of Paediatric Pulmonology and Immunology, Charité University Berlin, Germany; M. Amaral: Centre for Human Genetics, National Institute of Health Dr Ricardo Jorge, and Dept of Chemistry and Biochemistry, Faculty of Sciences, University of Lisbon, Lisbon, Portugal; S.M. Rowe: Dept of Medicine, University of Alabama, Birmingham, AL, USA; P. Middleton: Ludwig Engel Centre for Respiratory Research, Westmead Millennium Institute, Westmead, Australia; H. de Jonge: Depts of Paediatric Gastroenterology and Biochemistry, Erasmus University Medical Centre, Rotterdam, the Netherlands; I. Bronsveld and R.A. de Nooijer: University Medical Centre, Utrecht, the Netherlands; M. Wilschanski: Pediatric Gastroenterology Unit, Hadassah University Hospitals, Hebrew University Medical School, Jerusalem, Israel; P. Melotti: Centro Fibrosi Cistica, Azienda Ospedaliera Universitaria Integrata di Verona, Verona, Italy; I. Danner-Boucher: Laboratoire des Explorations Fonctionnelles and Service de Pneumologie, Hôpital G and R Laënnec, Nantes, France; S. Boerner: CF Center Cologne, University of Cologne, Cologne, Germany; I. Fajac: Service d'Explorations Fonctionnelles, Hôpital Cochin, Université Paris Descartes, Sorbonne Paris Cité, Paris, France; K. Southern: Dept of Women's and Children's Health, University of Liverpool, Liverpool, UK; A. Bot: Dept of Biochemistry, Sophia Children's Hospital, Erasmus MC, Rotterdam, the Netherlands; Y. de Rijke: Dept of Internal Medicine, Erasmus University Medical Centre, Rotterdam, the Netherlands; E. de Wachter: Dept of Pediatric Pulmonology and CF-Clinic, Universitair Ziekenhuis Brussel, Brussels, Belgium; T. Leal: Clinical Chemistry, Université Catholique de Louvain, Brussels, Belgium; M.J. Hug: University Medical Centre, 
Freiburg, Germany; G. Rault: Centre de Référence et de Compétence de la Mucoviscidose, Centre de Perharidy, Roscoff, France; T. Nguyen-Khoa: Depts of Biochemistry A and Paediatric Pulmonology, CRCM, Hôpital Necker-Enfants Malades AP-PH, Paris, France; C. Barreto: Dept of Paediatrics, Hospital de Santa Maria, Lisbon, Portugal; and I. SermetGaudelus: Hôpital Necker-Enfants Malades, Université Paris Descartes, INSERM U 845, Paris, France.

\section{REFERENCES}

1 De Gruttola VG, Clax P, DeMets DL, et al. Considerations in the evaluation of surrogate endpoints in clinical trials. Summary of a National Institutes of Health workshop. Control Clin Trials 2001; 22: 485-502.

2 Atkinson A, Colburn W, Gruttola VD, et al. Biomarkers and surrogate endpoints: preferred definitions and conceptual framework. Clin Pharmacol Ther 2001; 69: 89-95.

3 Fuchs HJ, Borowitz DS, Christiansen DH, et al. Effect of aerosolized recombinant human DNase on exacerbations of respiratory symptoms and on pulmonary function in patients with cystic fibrosis. The Pulmozyme Study Group. N Engl J Med 1994; 331: 637-642.

4 Ramsey BW, Pepe MS, Quan JM, et al. Intermittent administration of inhaled tobramycin in patients with cystic fibrosis. Cystic Fibrosis Inhaled Tobramycin Study Group. N Engl J Med 1999; 340: $23-30$.

5 Oermann CM, Retsch-Bogart GZ, Quittner AL, et al. An 18month study of the safety and efficacy of repeated courses of inhaled aztreonam lysine in cystic fibrosis. Pediatr Pulmonol 2010; 45: 1121-1134.

6 U.S. Department of Health and Human Services, Food and Drug Administration, Center for Drug Evaluation and Research, Center for Biologics Evaluation and Research, Center for Devices and Radiological Health. Guidance for Industry: Patient-reported Outcome Measures: Use in Medical Product Development to Support Labeling Claims. Health USDo, editor. Rockville, Food and Drug Administration, 2006.

7 Quittner A, Sawicki G, McMullen A, et al. Psychometric evaluation of the Cystic Fibrosis Questionnaire-Revised in a national sample. Qual Life Res, 2011: 1-12.

8 Committee for Medicinal Products for Human Use. Guideline on the Clinical Development of Medicinal Products for the Treatment of Cystic Fibrosis. London, European Medicines Agency, 2009.

9 Corey M, Edwards L, Levison $\mathrm{H}$, et al. Longitudinal analysis of pulmonary function decline in patients with cystic fibrosis. J Pediatr 1997; 131: 809-814.

10 Que C, Cullinan P, Geddes D. Improving rate of decline of FEV1 in young adults with cystic fibrosis. Thorax 2006; 61: 155-157.

11 Konstan MW, Wagener JS, Yegin A, et al. Design and powering of cystic fibrosis clinical trials using rate of FEV1 decline as an efficacy endpoint. J Cyst Fibros 2010; 9: 332-338.

12 Ramsey BW. Outcome measures for development of new therapies in cystic fibrosis: are we making progress and what are the next steps? Proc Am Thorac Soc 2007; 4: 367-369.

13 Mayer-Hamblett N, Ramsey BW, Kronmal RA. Advancing outcome measures for the new era of drug development in cystic fibrosis. Proc Am Thorac Soc 2007; 4: 370-377.

14 Rosenfeld M. An overview of endpoints for cystic fibrosis clinical trials: one size does not fit all. Proc Am Thorac Soc 2007; 4: 299-301.

15 Greenhalgh J, Long AF, Brettle AJ, et al. Reviewing and selecting outcome measures for use in routine practice. J Eval Clin Pract 1998; 4: 339-350.

16 Davies JC, Alton EW. Gene therapy for cystic fibrosis. Proc Am Thorac Soc 2010; 7: 408-414.

17 Wilschanski M, Yahav Y, Yaacov Y, et al. Gentamicin-induced correction of CFTR function in patients with cystic fibrosis and CFTR stop mutations. N Engl J Med 2003; 349: 1433-1441.
18 Kerem E, Hirawat S, Armoni S, et al. Effectiveness of PTC124 treatment of cystic fibrosis caused by nonsense mutations: a prospective phase II trial. Lancet 2008; 372: 719-727.

19 Sermet-Gaudelus I, Boeck KD, Casimir GJ, et al. Ataluren (PTC124) induces cystic fibrosis transmembrane conductance regulator protein expression and activity in children with nonsense mutation cystic fibrosis. Am J Respir Crit Care Med 2010; 182: 1262-1272.

20 Accurso FJ, Rowe SM, Clancy JP, et al. Effect of VX-770 in persons with cystic fibrosis and the G551D-CFTR mutation. N Engl J Med 2010; 363: 1991-2003.

21 Clancy JP, Rowe SM, Accurso FJ, et al. Results of a phase IIa study of VX-809, an investigational CFTR corrector compound, in subjects with cystic fibrosis homozygous for the F508delCFTR mutation. Thorax 2012; 67: 12-18.

22 Rosenstein BJ, Cutting GR. The diagnosis of cystic fibrosis: a consensus statement. Cystic Fibrosis Foundation Consensus Panel. J Pediatr 1998; 132: 589-595.

23 De Boeck K, Wilschanski M, Castellani C, et al. Cystic fibrosis: terminology and diagnostic algorithms. Thorax 2006; 61: 627-635.

24 Farrell PM, Rosenstein BJ, White TB, et al. Guidelines for diagnosis of cystic fibrosis in newborns through older adults: Cystic Fibrosis Foundation consensus report. J Pediatr 2008; 153: S4-S14.

25 De Boeck K, Derichs N, Fajac I, et al. New clinical diagnostic procedures for cystic fibrosis in Europe. J Cyst Fibros 2011; 10: Suppl. 2, S53-S66.

26 Green A. Guidelines for the Performance of the Sweat Test for the Investigation of Cystic Fibrosis in the UK. Royal College of Paediatrics and Child Health, 2003. Available from www.acb. org.uk/docs/sweat.pdf

27 Quinton PM. Chloride impermeability in cystic fibrosis. Nature 1983; 301: 421-422.

28 Knowles MR, Paradiso AM, Boucher RC. In vivo nasal potential difference: techniques and protocols for assessing efficacy of gene transfer in cystic fibrosis. Hum Gene Ther 1995; 6: 445-455.

29 Middleton PG, Caplen NJ, Gao X, et al. Nasal application of the cationic liposome DC-Chol:DOPE does not alter ion transport, lung function or bacterial growth. Eur Respir J 1994; 7: 442-445.

30 Boucher RC, Stutts MJ, Knowles MR, et al. $\mathrm{Na}^{+}$transport in cystic fibrosis respiratory epithelia. Abnormal basal rate and response to adenylate cyclase activation. J Clin Invest 1986; 78: 1245-1252.

31 Kunzelmann K, Kathofer S, Greger $\mathrm{R} \mathrm{Na}^{+}$and $\mathrm{Cl}^{-}$conductances in airway epithelial cells: increased $\mathrm{Na}^{+}$conductance in cystic fibrosis. Pflugers Arch 1995; 431: 1-9.

32 Reddy MM, Light MJ, Quinton PM. Activation of the epithelial $\mathrm{Na}^{+}$channel $(\mathrm{ENaC})$ requires CFTR $\mathrm{Cl}^{-}$channel function. Nature 1999; 402: 301-304.

33 Chen JH, Stoltz DA, Karp PH, et al. Loss of anion transport without increased sodium absorption characterizes newborn porcine cystic fibrosis airway epithelia. Cell 2010; 143: 911-923.

34 Itani OA, Chen JH, Karp PH, et al. Human cystic fibrosis airway epithelia have reduced $\mathrm{Cl}^{-}$conductance but not increased $\mathrm{Na}^{+}$ conductance. Proc Natl Acad Sci USA 2011; 108: 10260-10265.

35 Veeze HJ, Halley DJ, Bijman J, et al. Determinants of mild clinical symptoms in cystic fibrosis patients. Residual chloride secretion measured in rectal biopsies in relation to the genotype. J Clin Invest 1994; 93: 461-466.

36 De Jonge HR, Ballmann M, Veeze $\mathrm{H}$, et al. Ex vivo CF diagnosis by intestinal current measurements (ICM) in small aperture, circulating Ussing chambers. J Cyst Fibros 2004; 3: Suppl. 2, 159-163.

37 Derichs N, Sanz J, Von Kanel $\mathrm{T}$, et al. Intestinal current measurement for diagnostic classification of patients with questionable cystic fibrosis: validation and reference data. Thorax 2010; 65: 594-599.

38 Vermeulen F, Proesmans M, Feyaerts N, et al. Nasal potential measurements on the nasal floor and under the inferior turbinate: does it matter? Pediatr Pulmonol 2011; 46: 145-152. 
39 Jaron R, Yaakov Y, Rivlin J, et al. Nasal potential difference in non-classic cystic fibrosis-long term follow up. Pediatr Pulmonol 2008; 43: 545-549.

40 Yaakov Y, Kerem E, Yahav Y, et al. Reproducibility of nasal potential difference measurements in cystic fibrosis. Chest 2007; 132: 1219-1226.

41 Middleton PG, House HH. Measurement of airway ion transport assists the diagnosis of cystic fibrosis. Pediatr Pulmonol 2010; 45 789-795.

42 Ahrens RC, Standaert TA, Launspach J, et al. Use of nasal potential difference and sweat chloride as outcome measures in multicenter clinical trials in subjects with cystic fibrosis. Pediatr Pulmonol 2002; 33: 142-150.

43 Delmarco A, Pradal U, Cabrini G, et al. Nasal potential difference in cystic fibrosis patients presenting borderline sweat test. Eur Respir J 1997; 10: 1145-1149.

44 Hofmann $\mathrm{T}$, Bohmer $\mathrm{O}$, Huls $\mathrm{G}$, et al. Conventional and modified nasal potential-difference measurement in cystic fibrosis. Am J Respir Crit Care Med 1997; 155: 1908-1913.

45 Alton EW, Currie D, Logan-Sinclair R, et al. Nasal potential difference: a clinical diagnostic test for cystic fibrosis. Eur Respir J 1990; 3: 922-926.

46 Sermet-Gaudelus I, Renouil M, Fajac A, et al. In vitro prediction of stop-codon suppression by intravenous gentamicin in patients with cystic fibrosis: a pilot study. BMC Med 2007; 5: 5.

47 Clancy JP, Rowe SM, Bebok Z, et al. No detectable improvements in cystic fibrosis transmembrane conductance regulator by nasal aminoglycosides in patients with cystic fibrosis with stop mutations. Am J Respir Cell Mol Biol 2007; 37: 57-66.

48 Clancy JP, Bebok Z, Ruiz F, et al. Evidence that systemic gentamicin suppresses premature stop mutations in patients with cystic fibrosis. Am J Respir Crit Care Med 2001; 163: 1683-1692.

49 Wilschanski M, Famini C, Blau H, et al. A pilot study of the effect of gentamicin on nasal potential difference measurements in cystic fibrosis patients carrying stop mutations. Am J Respir Crit Care Med 2000; 161: 860-865.

50 Ramsey BW, Davies J, McElvaney NG, et al. A CFTR potentiator in patients with cystic fibrosis and the G551D mutation. $N$ Engl J Med 2011; 365: 1663-1672.

51 Aherns R. VX-770 in subjects 6 to 11 years with cystic fibrosis and the G551D-CFTR mutation. Pediatr Pulmonol 2011; 46: 283.

52 Boyle MP, Bell S, Konstan MW, et al. VX-809, an investigational CFTR corrector, in combination with VX-770, an investigational CFTR potentiator, in subjects with CF and homozygous for the F508del-CFTR mutation. Pediatric Pulmonology 2011; 46: 212-428.

53 Konstan MW, Davis PB, Wagener JS, et al. Compacted DNA nanoparticles administered to the nasal mucosa of cystic fibrosis subjects are safe and demonstrate partial to complete cystic fibrosis transmembrane regulator reconstitution. Hum Gene Ther 2004; 15: 1255-1269.

54 Noone PG, Hohneker KW, Zhou Z, et al. Safety and biological efficacy of a lipid-CFTR complex for gene transfer in the nasal epithelium of adult patients with cystic fibrosis. Mol Ther 2000; 1: 105-114.

55 Alton EW, Stern M, Farley R, et al. Cationic lipid-mediated CFTR gene transfer to the lungs and nose of patients with cystic fibrosis: a double-blind placebo-controlled trial. Lancet 1999; 353: 947-954

56 Zabner J, Cheng SH, Meeker D, et al. Comparison of DNA-lipid complexes and DNA alone for gene transfer to cystic fibrosis airway epithelia in vivo. J Clin Invest 1997; 100: 1529-1537.

57 Porteous DJ, Dorin JR, McLachlan G, et al. Evidence for safety and efficacy of DOTAP cationic liposome mediated CFTR gene transfer to the nasal epithelium of patients with cystic fibrosis. Gene Ther 1997; 4: 210-218.

58 Zabner J, Ramsey BW, Meeker DP, et al. Repeat administration of an adenovirus vector encoding cystic fibrosis transmembrane conductance regulator to the nasal epithelium of patients with cystic fibrosis. J Clin Invest 1996; 97: 1504-1511.

59 Gill DR, Southern KW, Mofford KA, et al. A placebo-controlled study of liposome-mediated gene transfer to the nasal epithelium of patients with cystic fibrosis. Gene Ther 1997; 4: 199-209.

60 Caplen NJ, Alton EW, Middleton PG, et al. Liposome-mediated CFTR gene transfer to the nasal epithelium of patients with cystic fibrosis. Nat Med 1995; 1: 39-46.

61 Hay JG, McElvaney NG, Herena J, et al. Modification of nasal epithelial potential differences of individuals with cystic fibrosis consequent to local administration of a normal CFTR cDNA adenovirus gene transfer vector. Hum Gene Ther 1995; 6: 1487-1496.

62 McCarty NA, Standaert TA, Teresi M, et al. A phase I randomized, multicenter trial of $\mathrm{CPX}$ in adult subjects with mild cystic fibrosis. Pediatr Pulmonol 2002; 33: 90-98.

63 Zeitlin PL, Boyle MP, Guggino WB, et al. A phase I trial of intranasal Moli1901 for cystic fibrosis. Chest 2004; 125: 143-149.

64 Rubenstein RC, Zeitlin PL. A pilot clinical trial of oral sodium 4-phenylbutyrate (Buphenyl) in deltaF508-homozygous cystic fibrosis patients: partial restoration of nasal epithelial CFTR function. Am J Respir Crit Care Med 1998; 157: 484-490.

65 Rodgers HC, Knox AJ. The effect of topical benzamil and amiloride on nasal potential difference in cystic fibrosis. Eur Respir J 1999; 14: 693-696.

66 Hofmann T, Senier I, Bittner P, et al. Aerosolized amiloride: dose effect on nasal bioelectric properties, pharmacokinetics, and effect on sputum expectoration in patients with cystic fibrosis J Aerosol Med 1997; 10: 147-158.

67 Hofmann T, Stutts MJ, Ziersch A, et al. Effects of topically delivered benzamil and amiloride on nasal potential difference in cystic fibrosis. Am J Respir Crit Care Med 1998; 157: 1844-1849.

68 Pyle LC, Fulton JC, Sloane PA, et al. Activation of the cystic fibrosis transmembrane conductance regulator by the flavonoid quercetin: potential use as a biomarker of DeltaF508 cystic fibrosis transmembrane conductance regulator rescue. Am J Respir Cell Mol Biol 2010; 43: 607-616.

69 Illek B, Fischer H. Flavonoids stimulate $\mathrm{Cl}$ conductance of human airway epithelium in vitro and in vivo. Am J Physiol 1998; 275: L902-L910.

70 Middleton PG, Pollard KA, Wheatley JR. Hypertonic saline alters ion transport across the human airway epithelium. Eur Respir J 2001; 17: 195-199.

71 Graham SM, Scott SN, Launspach J, et al. The effects of fluticasone propionate on nasal epithelial potential difference. Am I Rhinol 2002; 16: 145-149.

72 Smith SN, Middleton PG, Chadwick S, et al. The in vivo effects of milrinone on the airways of cystic fibrosis mice and human subjects. Am J Respir Cell Mol Biol 1999; 20: 129-134.

73 Boyle MP, Diener-West M, Milgram L, et al. A multicenter study of the effect of solution temperature on nasal potential difference measurements. Chest 2003; 124: 482-489.

74 Solomon GM, Konstan MW, Wilschanski M, et al. An international randomized multicenter comparison of nasal potential difference techniques. Chest 2010; 138: 919-928.

75 Alsuwaidan S, Li Wan Po A, Morrison G, et al. Effect of exercise on the nasal transmucosal potential difference in patients with cystic fibrosis and normal subjects. Thorax 1994; 49: 1249-1250.

76 Hebestreit A, Kersting U, Basler B, et al. Exercise inhibits epithelial sodium channels in patients with cystic fibrosis. Am J Respir Crit Care Med 2001; 164: 443-446.

77 Sermet-Gaudelus I, Girodon E, Roussel D, et al. Measurement of nasal potential difference in young children with an equivocal sweat test following newborn screening for cystic fibrosis. Thorax 2010; 65: 539-544. 
78 Sermet-Gaudelus I, Girodon E, Sands D, et al. Clinical phenotype and genotype of children with borderline sweat test and abnormal nasal epithelial chloride transport. Am J Respir Crit Care Med 2010; 182: 929-936.

79 Wilschanski M, Famini H, Strauss-Liviatan N, et al. Nasal potential difference measurements in patients with atypical cystic fibrosis. Eur Respir J 2001; 17: 1208-1215.

80 Berschneider HM, Knowles MR, Azizkhan RG, et al. Altered intestinal chloride transport in cystic fibrosis. FASEB J 1988; 2 : 2625-2629.

81 Hardcastle J, Hardcastle PT, Taylor CJ, et al. Failure of cholinergic stimulation to induce a secretory response from the rectal mucosa in cystic fibrosis. Gut 1991; 32: 1035-1039.

82 Hirtz S, Gonska T, Seydewitz $\mathrm{HH}$, et al. CFTR $\mathrm{Cl}^{-}$channel function in native human colon correlates with the genotype and phenotype in cystic fibrosis. Gastroenterology 2004; 127: 1085-1095.

83 Mall M, Bleich M, Kuehr J, et al. CFTR-mediated inhibition of epithelial $\mathrm{Na}^{+}$conductance in human colon is defective in cystic fibrosis. Am J Physiol 1999; 277: G709-G716.

84 Mall M, Wissner A, Seydewitz HH, et al. Defective cholinergic $\mathrm{Cl}^{-}$secretion and detection of $\mathrm{K}^{+}$secretion in rectal biopsies from cystic fibrosis patients. Am J Physiol Gastrointest Liver Physiol 2000; 278: G617-G624.

85 Taylor CJ, Baxter PS, Hardcastle J, et al. Failure to induce secretion in jejunal biopsies from children with cystic fibrosis. Gut 1988; 29: 957-962.

86 Taylor CJ, Hughes H, Hardcastle PT, et al. Genotype and secretory response in cystic fibrosis. Lancet 1992; 339: 67-68.

87 LeGrys VA, Yankaskas JR, Quittell LM, et al. Diagnostic sweat testing: the Cystic Fibrosis Foundation guidelines. J Pediatr 2007; 151: 85-89.

88 Legrys VA, McColley SA, Li Z, et al. The need for quality improvement in sweat testing infants after newborn screening for cystic fibrosis. J Pediatr 2010; 157: 1035-1037.

89 Southern KW, Noone PG, Bosworth DG, et al. A modified technique for measurement of nasal transepithelial potential difference in infants. J Pediatr 2001; 139: 353-358.
90 Clancy JP. Diagnosing cystic fibrosis in patients with nondiagnostic results: the case for intestinal current measurements. Thorax 2010; 65: 575-576.

91 Davies JC, Davies M, McShane D, et al. Potential difference measurements in the lower airway of children with and without cystic fibrosis. Am J Respir Crit Care Med 2005; 171: 1015-1019.

92 Wilschanski M, Miller LL, Shoseyov D, et al. Chronic ataluren (PTC124) treatment of nonsense mutation cystic fibrosis. Eur Respir J 2011; 38: 59-69.

93 Derichs N, Knoll J, Hyde R, et al. Preclinical evaluation of CFTR modulators in ex vivo human rectal tissue. Pediatric Pulmonology 2009; 44: 231.

94 Derichs N, Tran D, Namkung W, et al. Correction o $\Delta$ F508-CFTR in human airway epithelia and ex vivo rectal biopsies by s-cis-locked bithiazole corrector-29. J Cystic Fibros 2011; 10: Suppl. 1, S17.

95 Garcia MA, Yang N, Quinton PM. Normal mouse intestinal mucus release requires cystic fibrosis transmembrane regulator-dependent bicarbonate secretion. J Clin Invest 2009; 119: 2613-2622.

96 Amaral MD, Kunzelmann K. Molecular targeting of CFTR as a therapeutic approach to cystic fibrosis. Trends Pharmacol Sci 2007; 28: 334-341.

97 Wang F, Zeltwanger S, Hu S, et al. Deletion of phenylalanine 508 causes attenuated phosphorylation-dependent activation of CFTR chloride channels. J Physiol 2000; 524: 637-648.

98 US Food and Drug Administration. Food and Drug Administration Modernization Act. 1997.

99 Committee for Medicinal Products for Human Use. Guideline on Clinical Trials in Small Populations. London, European Medicines Agency, 2006.

100 Vertex Pharmaceuticals Inc. Interim Phase 2 Data Showed a Combination of VX-770 and VX-809 Improved Function of the Defective Protein that Causes Cystic Fibrosis in People With the Most Common Form of the Disease. http://investors.vrtx.com/ releasedetail.cfm?ReleaseID $=583683$ Date last updated: June 9, 2011. Date last accessed: November 6, 2012.

101 Dolmage TE, Hill K, Evans RA, et al. Has my patient responded? Interpreting clinical measurements such as the 6-minute-walk test. Am J Respir Crit Care Med 2011; 184: 642-646. 